\title{
PREPARATION OF 3-AMINO-3-DEOXY DERIVATIVES OF TREHALOSE AND SUCROSE AND THEIR ACTIVITIES
}

\author{
Naoki Asano, Katsumi Katayama, Masayoshi Takeuchi, \\ TADASH FuRUMOTO, YUKIHIKo KaMEDA \\ and KatsuHiKo Matsui \\ School of Pharmacy, Hokuriku University, \\ Kanazawa 920-11, Japan \\ (Received for publication October 26, 1988)
}

\begin{abstract}
The 3-keto derivatives of trehalose and sucrose respectively were prepared by a one-step enzymatic route using D-glucoside 3-dehydrogenase from Flavobacterium saccharophilum. Reductive amination of 3-ketotrehalose with ammonium acetate and sodium cyanoborohydride gave three compounds, 3-amino-3-deoxy- $\alpha$-D-allopyranosyl $\alpha$-D-glucopyranoside, 3amino-3-deoxy- $\alpha$-D-glucopyranosyl $\alpha$-D-glucopyranoside (3-trehalosamine) and 3-amino-3deoxy- $\alpha$-D-mannopyranosyl $\alpha$-D-glucopyranoside. From the reductive amination of 3 ketosucrose, 3-amino-3-deoxy- $\alpha$-D-allopyranosyl $\beta$-D-fructofuranoside and 3-amino-3-deoxy$\alpha$-D-glucopyranosyl $\beta$-D-fructofuranoside were obtained. The antibiotic and glycohydrolase inhibitory activities of these 3-amino-3-deoxy derivatives were determined.
\end{abstract}

D-Glucoside 3-dehydrogenase (EC 1.1.99.13) was first found in Agrobacterium tumefaciens, the agent for crown gall disease of several plants. This enzyme converts some hexopyranosides to the corresponding 3-keto compounds ${ }^{1 \sim 4)}$. The purification and characterization of this enzyme was reported by some researchers ${ }^{5 \sim 7}$. However, the D-glucoside 3-dehydrogenase of $A$. tumefaciens is an inducible enzyme and its activity rapidly decays on cessation of growth on cultivation. This feature limits the application of the bacteria as biosynthetic agents.

We recently found the D-glucoside 3-dehydrogenase in Flavobacterium saccharophilum which is involved in the degradation of the antifungal agents, validamycins, as the trigger enzyme in the C-N bond cleavage of validoxylamine $\mathrm{A}^{8 \sim 10}$. The D-glucoside 3-dehydrogenase in $F$. saccharophilum is a constitutive enzyme and approximately $80 \%$ of its activity is contained in the membrane fraction of this organism.

This paper describes the preparation of 3-ketotrehalose and 3-ketosucrose using a membrane fraction of $F$. saccharophilum, the reductive amination of these keto compounds with ammonium acetate and sodium cyanoborohydride, and the catalytic hydrogenation of the oximes obtained from the keto compounds. The antibiotic and glycohydrolase inhibitory activities of the 3-amino-3-deoxy disaccharides are also reported.

Preparation of 3-Ketotrehalose and 3-Ketosucrose

3-Ketotrehalose and 3-ketosucrose were prepared by the one-step enzymatic route using a membrane fraction of $F$. saccharophilum. The resulting incubation mixture of a substrate $(1 \mathrm{~g})$ and a membrane fraction was centrifuged and the supernatant was applied to a column of activated carbon and eluted with $50 \% \mathrm{MeOH}$. The concentrate of the eluate was then applied to a column of Dowex 50W-X8 $\left(\mathrm{Ca}^{2+}\right)$. The column was developed with water to give 3-ketotrehalose (550 mg) or 3-ketosucrose ( $470 \mathrm{mg}$ ). The structure of the 3-keto compounds were confirmed by ${ }^{1} \mathrm{H}$ and ${ }^{18} \mathrm{C}$ NMR data. 
Fig. 1. Structures of 3-amino-3-deoxy disaccharides.

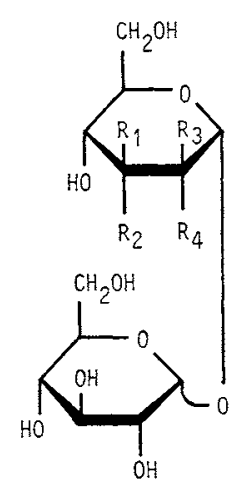

T-I T-II T-III
$\mathrm{R}_{1}=\mathrm{R}_{3}=\mathrm{H} \quad \mathbf{R}_{2}=\mathrm{NH}_{2} \quad \mathrm{R}_{4}=\mathrm{OH}$

$$
\mathbf{R}_{1}=\mathrm{NH}_{2} \quad \mathbf{R}_{2}=\mathbf{R}_{3}=\mathbf{H} \quad \mathbf{R}_{4}=\mathrm{OH}
$$$$
\mathrm{R}_{1}=\mathrm{NH}_{2} \quad \mathrm{R}_{2}=\mathrm{R}_{4}=\mathrm{H} \quad \mathrm{R}_{3}=\mathrm{OH}
$$

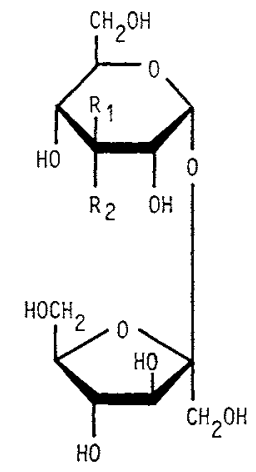

S-I $\quad \mathrm{R}_{1}=\mathrm{H} \quad \mathrm{R}_{2}=\mathrm{NH}_{2}$ S-II $\quad R_{1}=\mathrm{NH}_{2} \quad \mathrm{R}_{2}=\mathrm{H}$

Reductive Amination of 3-Keto Compounds

The reductive amination of 3-ketotrehalose $(1 \mathrm{~g})$ with ammonium acetate and sodium cyanoborohydride in $\mathrm{MeOH}$ gave a mixture of compounds T-I, T-II and T-III. The mixture was purified by the column chromatography on activated carbon and Amberlite CG-50 $\left(\mathrm{NH}_{4}^{+}\right)$. The succeeding chromatography on Dowex 1-X2 $\left(\mathrm{OH}^{-}\right)$with water gave homogeneous compounds T-I (150 mg), T-II $(65 \mathrm{mg})$ and T-III ( $28 \mathrm{mg})$, respectively. The reductive amination of 3-ketosucrose yielded compounds S-I and S-II, which were separated from the reaction mixture by column chromatography on CM-Sephadex $\mathrm{C}-25\left(\mathrm{NH}_{4}{ }^{+}\right)$. Compounds S-I and S-II were isolated as compound S-I hydrochloride $(160 \mathrm{mg})$ and compound S-II hydrochloride $(20 \mathrm{mg})$ by Dowex $1-\mathrm{X} 2\left(\mathrm{OH}^{-}\right)$chromatography with $0.2 \mathrm{~N}$ hydrochloric acid because compounds S-I and S-II were rigidly retained on a Dowex 1-X2 $\left(\mathrm{OH}^{-}\right)$ column.

Catalytic hydrogenation of the oxime obtained by treatment of 3-ketotrehalose $(500 \mathrm{mg})$ with hydroxylamine in $\mathrm{MeOH}$ stereoselectively formed compound T-I $(240 \mathrm{mg})$. In the case of 3-ketosucrose, the desired 3-amino-3-deoxy disaccharide was not obtained using similar techniques because the glycosidic bond of the oxime derivative was cleaved by catalytic hydrogenation.

\section{Structure Determination of 3-Amino-3-deoxy Derivatives}

Compound T-I or S-I was heated under reflux with Amberlyst 15 in $\mathrm{MeOH}$ for 40 hours. The hexosamine moiety was adsorbed on the ion-exchange resin and eluted from the resin with concentrated $\mathrm{NH}_{4} \mathrm{OH}-\mathrm{MeOH}(14: 86)$. The eluate was chromatographed on CM-Sephadex $\mathrm{C}-25\left(\mathrm{NH}_{4}{ }^{+}\right)$ and Dowex 1-X2 $\left(\mathrm{OH}^{-}\right)$to give two products. They were identified as methyl 3-amino-3-deoxy- $\alpha-$ D-allopyranoside and 3-amino-1,6-anhydro-3-deoxy- $\beta$-D-allopyranose by direct comparison of their ${ }^{1} \mathrm{H},{ }^{13} \mathrm{C}$ NMR spectra and GC-MS analysis with those of authentic samples. Thus, the structures of compounds T-I and S-I were determined to be 3-amino-3-deoxy- $\alpha$-D-allopyranosyl $\alpha$-D-glucopyranoside and 3-amino-3-deoxy- $\alpha$-D-allopyranosyl $\beta$-D-fructofuranoside, respectively. In the same manner, the structures of compounds T-II and S-II were confirmed to be 3-amino-3-deoxy- $\alpha$-D-glucopyranosyl $\alpha$-D-glucopyranoside (3-trehalosamine) ${ }^{11,122}$ and 3-amino-3-deoxy- $\alpha$-D-glucopyranosyl $\beta$-D-fructofuranoside (designated as 3-sucrosamine), respectively, since the methanolysis of these compounds 
gave methyl 3-amino-3-deoxy- $\alpha$-D-glucopyranoside and its $\beta$-anomer. The structure of compound T-III was determined to be 3-amino-3-deoxy- $\alpha$-D-mannopyranosyl $\alpha$-D-glucopyranoside since its methanolysis gave only methyl 3-amino-3-deoxy- $\alpha$-D-mannopyranoside.

\section{Biological Properties}

2-Trehalosamine ${ }^{13)}$, 3-trehalosamine ${ }^{12)}$, 4-trehalosamine ${ }^{14)}$ and mannosyl glucosaminide ${ }^{15}$ have so far been reported as naturally occurring 1-1 linked aminodisaccharide antibiotics. They are all $\alpha, \alpha$-glycosides of an aminosugar and a neutral sugar. Recently, the only example of $\alpha, \beta$-glycoside of two aminosugars, 3,3'-neotrehalosadiamine, has been reported ${ }^{16)}$. The synthetic 3,3'-diamino-3,3'dideoxy disaccharide, 3-amino-3-deoxy- $\alpha$-D-mannopyranosyl 3-amino-3-deoxy- $\alpha$-D-mannopyranoside $^{17)}$, possesses a remarkable inhibitory activity against Mycobacterium tuberculosis and Mycobacterium avium, whereas the synthetic 6 -trehalosamine ${ }^{18)}$ is inactive.

The prepared 3-amino-3-deoxy disaccharides, except compound S-I, showed weak antibacterial activity against some bacterial species by the cylinder-plate assay method (Table 1). In particular, it is noteworthy that 3-sucrosamine (S-II) is no less active against Staphylococcus aureus and Bacillus subtilis than 3-trehalosamine (T-II). Compounds T-I and T-III showed weak antibacterial activity against Escherichia coli, while 3-trehalosamine and 3-sucrosamine were inactive.

Table 1. Cylinder-plate assay of 3-amino-3-deoxy disaccharides.

\begin{tabular}{lccccc}
\hline & \multicolumn{5}{c}{ Inhibition zone (mm) at 1 mg/ml } \\
\cline { 2 - 6 } Organism & \multicolumn{5}{c}{ Compounds } \\
\cline { 2 - 6 } & T-I & T-II & T-III & S-I & S-II \\
\hline Staphylococcus aureus FDA 209P & - & 27 & - & - & 23 \\
Bacillus subtilis PCI 219 & 15 & 16 & 11 & - & 23 \\
Mycobacterium smegmatis ATCC 607 & - & - & - & - & - \\
Escherichia coli K-12 & 19 & - & 11 & - & - \\
Pseudomonas aeruginosa IFO 1313 & - & - & - & - & - \\
Saccharomyces cerevisiae IFO 0209 & - & - & - & - & - \\
Candida albicans IFO 0583 & - & - & - & - & - \\
\hline
\end{tabular}

-: No inhibition zone.

Table 2. Inhibitory activity of 3-amino-3-deoxy disaccharides against glycohydrolases.

\begin{tabular}{|c|c|c|c|c|c|c|}
\hline \multirow{3}{*}{$\begin{array}{l}\text { Enzyme } \\
\text { (origin) }\end{array}$} & \multicolumn{6}{|c|}{$\mathrm{IC}_{50}(\mathrm{M})$} \\
\hline & \multicolumn{5}{|c|}{ Compounds } & \multirow{2}{*}{ 2-Trehalosamine } \\
\hline & T-I & T-II & T-III & S-I & S-II & \\
\hline \multicolumn{7}{|l|}{ Invertase } \\
\hline Porcine & - & - & - & $3.1 \times 10^{-4}$ & - & - \\
\hline Baker's yeast & - & - & - & $5.6 \times 10^{-4}$ & - & - \\
\hline Candida utilis & - & - & - & $7.8 \times 10^{-4}$ & - & - \\
\hline \multicolumn{7}{|l|}{ Maltase } \\
\hline Porcine & - & - & - & - & - & - \\
\hline Baker's yeast & - & - & - & - & - & - \\
\hline \multicolumn{7}{|l|}{ Trehalase } \\
\hline Porcine & - & - & - & - & - & - \\
\hline Baker's yeast & - & - & - & - & - & $7.2 \times 10^{-5}$ \\
\hline Spodoptera litura & - & - & - & - & $7.4 \times 10^{-4}$ & - \\
\hline
\end{tabular}

-: No activity at $10^{-3} \mathrm{M}$. 
The 3-amino-3-deoxy disaccharides prepared from trehalose showed no inhibitory activity against any glycohydrolases tested. Compounds S-I and S-II showed weak inhibitory activity against various invertases and an insect trehalase, respectively (Table 2).

\section{Experimental}

MP's were determined with a Yamato MP-21 apparatus and are uncorrected. Optical rotations were measured with a Jasco digital polarimeter DIP-4. ${ }^{1} \mathrm{H}$ NMR spectra were recorded with a Jeol JNM-FX100 spectrometer (at $100 \mathrm{MHz}$ ). Chemical shifts are reported in ppm from internal sodium 3-(trimethylsilyl)propionate (TSP, in $\mathrm{D}_{2} \mathrm{O}$ ) or TMS (in $\mathrm{CDCl}_{3}$ or $\mathrm{C}_{8} \mathrm{D}_{6}$ ). ${ }^{13} \mathrm{C}$ NMR spectra were recorded with Jeol JNM-FX100 spectrometer at $25.0 \mathrm{MHz}$. The trimethylsilyl derivatives of hexosamines were analyzed with GC-MS (Jeol JMS-DX300 JMA-DA5000). TLC was performed on precoated Kieselgel $\mathrm{F}_{254}$ plates (Merck) with $\mathrm{PrOH}-\mathrm{AcOH}-\mathrm{H}_{2} \mathrm{O}(4: 1: 1)$, unless otherwise specified.

\section{3-Aminohexoses}

Methyl 3-amino-3-deoxy- $\beta$-D-glucopyranoside and methyl 3-amino-3-deoxy- $\alpha$-D-mannopyranoside were purchased from Lucerna-Chem AG.; 3-amino-3-deoxy-D-glucose from Calbiochem-Behring Corp.; and 3-acetamido-3-deoxy-D-allopyranose from Sigma Chemical Company. Methyl 3-amino3-deoxy- $\alpha$-D-glucopyranoside ${ }^{19)}$ and methyl 3-amino-3-deoxy- $\beta$-D-glucopyranoside were prepared by methanolysis of 3-amino-3-deoxy-D-glucose. 3-Amino-1,6-anhydro-3-deoxy- $\beta$-D-allopyranose and methyl 3-amino-3-deoxy- $\alpha$-D-allopyranoside were prepared by methanolysis of 3-acetamide-3-deoxyD-allopyranose.

\section{GC-MS Analysis of Aminohexose Trimethylsilyl Derivatives}

Approxymately $1 \mathrm{mg}$ of the aminohexoses in a silicone-capped small tube were dissolved into $100 \mu \mathrm{l}$ of pyridine. Bis(trimethylsilyl)acetamide $100 \mu \mathrm{l}$ and trimethylchlorosilane $50 \mu \mathrm{l}$ were added. The tube was heated at $75^{\circ} \mathrm{C}$ for 20 minutes. Each sample was analyzed on a glass column $(0.26 \times$ $200 \mathrm{~cm}$ ) with $7 \%$ silicone OV-17 on Chromosorb W AW DMCS. The reaction was performed at $175^{\circ} \mathrm{C}$. The GC-MS analysis of aminohexose $N, O$-trimethylsilyl derivatives resulted in the following retention times $\left(\mathrm{t}_{\mathrm{R}}\right)$ and $m / z$ peaks;

3-Amino-1,6-anhydro-3-deoxy- $\beta$-D-allopyranose: $t_{\mathrm{R}} 4.4$ minutes, $m / z 377(\mathrm{M})^{+}$.

Methyl 3-amino-3-deoxy- $\alpha$-D-allopyranoside: $\mathrm{t}_{\mathrm{R}} 6.7$ minutes, $m / z 481(\mathrm{M})^{+}$.

Methyl 3-amino-3-deoxy- $\alpha$-D-glucopyranoside: $\mathrm{t}_{\mathrm{R}} 11.1$ minutes, $m / z 481(\mathrm{M})^{+}$.

Methyl 3-amino-3-deoxy- $\beta$-D-glucopyranoside: $\mathrm{t}_{\mathrm{R}} 10.2$ minutes, $m / z 481(\mathrm{M})^{+}$.

Methyl 3-amino-3-deoxy- $\alpha$-D-mannopyranoside: $\mathrm{t}_{\mathrm{R}} 6.2$ minutes, $m / z 481(\mathrm{M})^{+}$.

Preparation of 3-Ketotrehalose and 3-Ketosucrose

A mixture of trehalose $(1 \mathrm{~g})$ or sucrose $(1 \mathrm{~g})$ and the membrane fraction ${ }^{8,9}$ ) obtained from $150 \mathrm{~g}$ wet cells of $F$. saccharophilum in $0.05 \mathrm{M}$ acetate buffer $(\mathrm{pH} 5.5,100 \mathrm{ml})$ containing $1 \mathrm{mM}$ EDTA were incubated at $37^{\circ} \mathrm{C}$ for 9 hours with shaking. The incubation mixture was centrifuged and the supernatant was applied to a column of activated carbon $(200 \mathrm{ml})$ and eluted with $50 \% \mathrm{MeOH}$. The concentrate was then applied to a column of Dowex $50 \mathrm{~W}-\mathrm{X} 8\left(\mathrm{Ca}^{2+}, 450 \mathrm{~mJ}\right)$. The column was developed with water to give 3-ketotrehalose $(550 \mathrm{mg})$ or 3-ketosucrose $(470 \mathrm{mg})$.

$\alpha$-D-Glucopyranosyl $\alpha$-D-ribo-3-Hexosulopyranoside (3-Ketotrehalose): Rf $0.43 ;[\alpha]_{\mathrm{D}}^{20}+147.1^{\circ}$ $\left.(c) 1, \mathrm{H}_{2} \mathrm{O}\right)$; ${ }^{1} \mathrm{H}$ NMR $\left(\mathrm{D}_{2} \mathrm{O}\right) \delta 3.32 \sim 4.16(9 \mathrm{H}), 4.43(1 \mathrm{H}, \mathrm{dd}, J=1.5$ and $9.5 \mathrm{~Hz}, 4-\mathrm{H}), 4.68(1 \mathrm{H}$, dd, $J=1.5$ and $4.6 \mathrm{~Hz}, 2-\mathrm{H}), 5.17\left(1 \mathrm{H}, \mathrm{d}, J=3.2 \mathrm{~Hz}, 1^{\prime}-\mathrm{H}\right), 5.56(1 \mathrm{H}, \mathrm{d}, J=4.6 \mathrm{~Hz}, 1-\mathrm{H}) ;{ }^{13} \mathrm{C}$ NMR $\left(\mathrm{D}_{2} \mathrm{O}\right)$ $\delta 63.2\left(\mathrm{t}, \mathrm{C}-6, \mathrm{C}-6^{\prime}\right), 72.3\left(\mathrm{~d}, \mathrm{C}-4^{\prime}\right), 73.7\left(\mathrm{~d}, \mathrm{C}-2^{\prime}\right), 74.4\left(\mathrm{~d}, \mathrm{C}-5^{\prime}\right), 75.1$ (d, C-4, C-3'), $76.6(\mathrm{~d}, \mathrm{C}-2), 78.0$ (d, C-5), $96.5(d, C-1$ ), 99.0 (d, C-1), 209.8 (s, C-3).

$\beta$-D-Fructofranosyl $\alpha$-D-ribo-3-Hexosulopyranoside (3-Ketosucrose): $\operatorname{Rf} 0.46 ;[\alpha]_{D}^{20}+55.0^{\circ}(c 1$, $\left.\mathrm{H}_{2} \mathrm{O}\right)$; ${ }^{1} \mathrm{H}$ NMR $\left(\mathrm{D}_{2} \mathrm{O}\right) \delta 3.63 \sim 4.27(10 \mathrm{H}), 4.45(1 \mathrm{H}$, dd, $J=1.5$ and $9.5 \mathrm{~Hz}, 4-\mathrm{H}), 4.61(1 \mathrm{H}, \mathrm{dd}, J=$ 1.5 and $4.6 \mathrm{~Hz}, 2-\mathrm{H}), 5.79(1 \mathrm{H}, \mathrm{d}, J=4.6 \mathrm{~Hz}, 1-\mathrm{H}) ;{ }^{13} \mathrm{C}$ NMR $\left(\mathrm{D}_{2} \mathrm{O}\right) \delta 63.0(\mathrm{t}, \mathrm{C}-6), 63.9\left(\mathrm{t}, \mathrm{C}-1^{\prime}\right)$, 65.1 (t, C-6'), 74.1 (d, C-4), 76.6 (d, C-2, C-4'), 78.2 (d, C-5), 78.9 (d, C-3'), $84.2\left(\mathrm{~d}, \mathrm{C}-5^{\prime}\right), 97.7$ (d, C-1), $106.8(\mathrm{~s}, \mathrm{C}-2), 210.2(\mathrm{~s}, \mathrm{C}-3)$. 
Reductive Amination of 3-Keto Compounds

Method A: To a solution of 3-ketotrehalose $(1 \mathrm{~g})$ and $\mathrm{CH}_{3} \mathrm{COONH}_{4}(3 \mathrm{~g})$ in $\mathrm{MeOH}(20 \mathrm{ml})$, $\mathrm{NaBH}_{3} \mathrm{CN}(1.5 \mathrm{~g})$ and $\mathrm{HCl}(2 \mathrm{~N}, 0.6 \mathrm{ml})$ were added, and the solution was stirred for 16 hours at room temperature. The mixture was concentrated, dissolved in water $(200 \mathrm{ml})$, and then applied to a column of activated carbon $(200 \mathrm{ml})$. The column was washed with water and eluted with $50 \% \mathrm{MeOH}$. The eluate was concentrated and chromatographed on a column of Amberlite CG-50 $\left.\mathrm{NH}_{4}^{+}, 100 \mathrm{ml}\right)$ with $0.1 \mathrm{~N} \mathrm{NH}_{4} \mathrm{OH}$. Finally, the eluate was chromatographed on a column of Dowex 1-X2 $\left(\mathrm{OH}^{-}\right.$, $170 \mathrm{ml})$ with water to give homogeneous compounds T-I $(150 \mathrm{mg}), \mathrm{T}-\mathrm{II}(65 \mathrm{mg})$ and T-III $(28 \mathrm{mg})$, respectively.

Compound T-I: Rf $0.33 ;[\alpha]_{\mathrm{D}}^{20}+184.0^{\circ}\left(c 0.5, \mathrm{H}_{2} \mathrm{O}\right) ;{ }^{1} \mathrm{H}$ NMR $\left(\mathrm{D}_{2} \mathrm{O}\right) \delta 5.14(1 \mathrm{H}, \mathrm{d}, J=3.4 \mathrm{~Hz}$, anomeric proton), $5.18\left(1 \mathrm{H}, \mathrm{d}, J=3.4 \mathrm{~Hz}\right.$, anomeric proton); ${ }^{13} \mathrm{C}$ NMR ( $\left.\mathrm{D}_{2} \mathrm{O}, \mathrm{pD} 2.0\right) \delta 57.1(\mathrm{~d}, \mathrm{C}-3)$, 63.0 (t, C-6), 63.2 (t, C-6'), 64.8 (d, C-4), 66.5 (d, C-2), 70.2 (d, C-5), 72.1 (d, C-4'), 73.1 (d, C-2'), 75.1 (d, C-5'), 75.5 (d, C-3'), 95.8 (d, C-1), 96.4 (d, C-1').

Compound T-II: $\quad \operatorname{Rf} 0.30 ;[\alpha]_{\mathrm{D}}^{20}+207.6^{\circ}\left(c 0.5, \mathrm{H}_{2} \mathrm{O}\right)\left(\right.$ literature $12,+161^{\circ}\left(c 13.7, \mathrm{H}_{2} \mathrm{O}\right)$ ); ${ }^{1} \mathrm{H}$ $\operatorname{NMR}\left(\mathrm{D}_{2} \mathrm{O}\right) \delta 5.15(1 \mathrm{H}, \mathrm{d}, J=3.7 \mathrm{~Hz}$, anomeric proton), $5.19(1 \mathrm{H}, \mathrm{d}, J=3.7 \mathrm{~Hz}$, anomeric proton); ${ }^{13} \mathrm{C}$ NMR $\left(\mathrm{D}_{2} \mathrm{O}, \mathrm{pD} 1.7\right) \delta 57.6(\mathrm{~d}, \mathrm{C}-3), 62.6(\mathrm{t}, \mathrm{C}-6), 63.3\left(\mathrm{t}, \mathrm{C}-6^{\prime}\right), 68.6(\mathrm{~d}, \mathrm{C}-4), 70.4(\mathrm{~d}, \mathrm{C}-2), 72.4$ (d, C-4'), 73.7 (d, C-2'), 74.7 (d, C-5), 75.0 (d, C-5), 75.2 (d, C-3'), 95.0 (d, C-1), 96.2 (d, C-1').

Compound T-III: Rf $0.30 ;[\alpha]_{D}^{20}+145.0^{\circ}\left(c 0.5, \mathrm{H}_{2} \mathrm{O}\right) ;{ }^{1} \mathrm{H}$ NMR $\left(\mathrm{D}_{2} \mathrm{O}\right) \delta 5.08(1 \mathrm{H}, \mathrm{d}, J=1.7 \mathrm{~Hz}$, anomeric proton), $5.19\left(1 \mathrm{H}, \mathrm{d}, J=3.4 \mathrm{~Hz}\right.$, anomeric proton); ${ }^{18} \mathrm{C} \mathrm{NMR}\left(\mathrm{D}_{2} \mathrm{O}, \mathrm{pD} 1.9\right) \delta 55.8(\mathrm{~d}, \mathrm{C}-3)$, $63.0(\mathrm{t}, \mathrm{C}-6), 63.3\left(\mathrm{t}, \mathrm{C}-6^{\prime}\right), 65.9$ (d, C-2), 69.5 (d, C-4), 72.3 (d, C-4'), 73.5 (d, C-2'), 75.3 (d, C-5, C-5), 75.6 (d, C-3'), $96.3\left(\mathrm{~d}, \mathrm{C}-1^{\prime}\right), 96.7$ (d, C-1).

3-Ketosucrose $(1 \mathrm{~g})$ was treated with the same manner mentioned above. The reaction mixture was purified on a column of activated carbon $(200 \mathrm{ml})$ and Amberlite CG-50 $\left(\mathrm{NH}_{4}^{+}, 100 \mathrm{ml}\right)$. The eluate from Amberlite CG-50 was chromatographed on a CM-Sephadex C-25 $\left(\mathrm{NH}_{4}{ }^{+}, 100 \mathrm{ml}\right)$ with $0.02 \mathrm{~N} \mathrm{NH}_{4} \mathrm{OH}$ to resolve two fractions. Each fraction was chromatographed on a column of Dowex $1-\mathrm{X} 2\left(\mathrm{OH}^{-}, 50 \mathrm{ml}\right)$. The column was washed with water and then eluted with $0.2 \mathrm{~N} \mathrm{HCl}$. The eluate was concentrated and then lyophilized to give compound S-I hydrochloride (160 $\mathrm{mg}$ ) or compound S-II hydrochloride $(20 \mathrm{mg})$.

Compound S-I Hydrochloride: Rf $0.40 ;[\alpha]_{1}^{20}+41.1^{\circ}\left(c 0.5, \mathrm{H}_{2} \mathrm{O}\right) ;{ }^{1} \mathrm{H}$ NMR $\left(\mathrm{D}_{2} \mathrm{O}\right) \delta 5.46(1 \mathrm{H}$, $\mathrm{d}, J=3.4 \mathrm{~Hz}$, anomeric proton); ${ }^{13} \mathrm{C} \mathrm{NMR}\left(\mathrm{D}_{2} \mathrm{O}\right) \delta 57.2$ (d, C-3), 62.7 (t, C-6), 64.5 (d, C-4), 64.5 (t, C-1'), 66.4 (d, C-2), 70.4 (d, C-5), 76.4 (d, C-4'), 79.4 (d, C-3'), 84.3 (d, C-5'), 94.3 (d, C-1), 106.9 (s, C-2').

Compound S-II Hydrochloride: Rf 0.36; $[\alpha]_{\mathrm{D}}^{20}+173.0^{\circ}\left(c 0.5, \mathrm{H}_{2} \mathrm{O}\right) ;{ }^{1} \mathrm{H}$ NMR $\left(\mathrm{D}_{2} \mathrm{O}\right) \delta 5.45$ $\left(1 \mathrm{H}, \mathrm{d}, J=3.7 \mathrm{~Hz}\right.$, anomeric proton); ${ }^{13} \mathrm{C}$ NMR $\left(\mathrm{D}_{2} \mathrm{O}\right) \delta 57.7$ (d, C-3), $62.3(\mathrm{t}, \mathrm{C}-6), 64.1$ (t, C-1'), $65.1\left(\mathrm{t}, \mathrm{C}-6^{\prime}\right), 68.3$ (d, C-4), 70.5 (d, C-2), 75.0 (d, C-5), 76.7 (d, C-4'), 79.2 (d, C-3'), 84.2 (d, C-5'), 93.9 (d, C-1), 106.6 (s, C-2').

Method B: A solution of 3-ketotrehalose $(500 \mathrm{mg})$ and hydroxylamine hydrochloride $(200 \mathrm{mg})$ in $\mathrm{MeOH}$ was stirred at room temperature for 3 hours. The reaction mixture was hydrogenated in the presence of $\mathrm{PtO}_{2}$ at atmospheric pressure for 6 hours. The filtrate was concentrated and chromatographed on a column of Dowex $1-\mathrm{X} 2\left(\mathrm{OH}^{-}, 200 \mathrm{ml}\right)$. The column was eluted with water to give compound T-I $(240 \mathrm{mg})$ as the sole product.

In the case of 3-ketosucrose, the desired 3-amino-3-deoxy derivative was not formed using the same method mentioned above.

\section{Assay of Glycohydrolase Inhibitory Activity}

Invertase (Candida utilis and baker's yeast) and maltase (baker's yeast) were purchased from Sigma Chemical Company. Invertase, maltase and trehalase from procine sources were prepared from small intestine mucosa according to the method of DAHLQVIST ${ }^{20}$. The trehalases of baker's yeast $^{21)}$ and insect ${ }^{22)}$ (Spodoptera litura) were prepared as described in the literature. The inhibitory activity was determined by incubating an enzyme solution $(125 \mu 1)$ with a $0.5-\mathrm{M}$ substrate solution $(50 \mu \mathrm{l})$ and an inhibitor solution $(200 \mu \mathrm{l})$ in $0.05 \mathrm{M}$ phosphate buffer $(\mathrm{pH} 6.0$, in a final volume of 500 $\mu l)$ at $37^{\circ} \mathrm{C}$ for 15 minutes and by measuring the amount of released $\mathrm{D}$-glucose by the glucose oxidase 
method using the commercially available Glucose B-Test Wako kit (Wako Pure Chemical Industries, Ltd.).

\section{References}

1) Bernaerts, M. J. \& J. De Ley: 3-Ketoglucosides; new intermediates in the bacterial catabolism of disaccharides. Biochim. Biophys. Acta 30:661 663, 1958

2) FukUi, S. \& R. M. HochSTER: Conversion of disaccharides to the corresponding glycoside-3-uloses by intact cells of Agrobacterium tumefaciens. Can. J. Biochem. Physiol. 41: 2363 2371, 1963

3) Kurowski, W. M. \& S. J. PIRT: The iron requirement of Agrobacterium tumefaciens for growth and 3-ketosucrose production. The removal of iron from solutions by Serrz filters. J. Gen. Microbiol. 68: $65 \sim 69,1971$

4) Tyler, D. D. \& L. K. Nakamura: Conditions for production of 3-ketomaltose from Agrobacterium tumefaciens. Appl. Microbiol. 21: 175 182, 1971

5) Grebner, E. E. \& D. S. Feingold: D-Aldohexopyranoside dehydrogenase of Agrobacterium tumefaciens. Biochem. Biophys. Res. Commun. 19: 37 42, 1965

6) Hayano, K. \& S. Fukui: Purification and properties of 3-ketosucrose-forming enzyme from the cells of Agrobacterium tumefaciens. J. Biol. Chem. 242: 3665 3672, 1967

7) Van Beeumen, J. \& J. De LeY: Hexopyranoside: cytochrome c oxidoreductase from Agrobacterium tumefaciens. Eur. J. Biochem. 6: 331 343, 1968

8) Asano, N.; M. TAKeUchi, K. Ninomiya, Y. Kameda \& K. Matsur: Microbial degradation of validamycin A by Flavobacterium saccharophilum. Enzymatic cleavage of $\mathrm{C}-\mathrm{N}$ linkage in validoxylamine A. J. Antibiotics 37: 859 867, 1984

9) Takeuchi, M.; K. Ninomiya, K. Kawabata, N. Asano, Y. Kameda \& K. Matsui: Purification and properties of glucoside 3-dehydrogenase from Flavobacterium saccharophilum. J. Biochem. 100: 1049 1055,1986

10) Takeuchi, M.; N. Asano, Y. Kameda \& K. Matsui: Purification and properties of soluble D-glucoside 3-dehydrogenase from Flavobacterium saccharophilum. Agric. Biol. Chem. 52: 1905 1912, 1988

11) BAER, H. H. \& A. J. BeLl: The synthesis of 3-amino-3-deoxy- $\alpha$-D-glucopyranosyl $\alpha$-D-glucopyranoside (3-amino-3-deoxy- $\alpha, \alpha$-trehalose). Carbohydr. Res. 75: 175 184, 1979

12) Dolak, L. A.; T. M. CASTle \& A. L. LaboRdE: 3-Trehalosamine, a new disaccharide antibiotic. J. Antibiotics 33: 690 694, 1980

13) ARCAmone, F. \& B. Bizioli: Isolation and constitution of trehalosamine, a new aminosugar from a Streptomyces tomyces. Gazz. Chim. Ital. 87: 896 902, 1957

14) Naganawa, H.; N. Usui, T. Takita, M. Hamada, K. Maeda \& H. Umezawa: 4-Amino-4-deoxy- $\alpha, \alpha-$ trehalose, a new metabolite of a streptomyces. J. Antibiotics 27: 145 146, 1974

15) Uramoto, M.; N. ŌTake \& H. Yonehara: Mannosyl glucosaminide, a new antibiotic. J. Antibiotics, Ser. A 20: 236 237, 1967

16) Tsuno, T.; C. Ikeda, K. Numata, K. Tomita, M. Konishi \& H. Kawaguchi: 3,3'-Neotrehalosadiamine (BMY-28251), a new aminosugar antibiotic. J. Antibiotics 39: 1001 1003, 1986

17) Baer, H. H.; S. Radatus \& J. Defaye: A new synthesis of 3-amino-3-deoxy- $\alpha$-D-mannopyranosyl 3amino-3-deoxy- $\alpha$-D-mannopyranoside, a potent antituberculous agent. Can. J. Chem. 63: 440 444, 1985

18) Hanessian, S. \& P. LAVAlléE: Synthesis of 6-amino-6-deoxy- $\alpha, \alpha$-trehalose: A positional isomer of trehalosamine. J. Antibiotics 25:683 684, 1972

19) Koch, K. F.; J. A. RhOAdes, E. W. Hagaman \& E. Wenkert: Carbon-13 nuclear magnetic resonance spectral analysis of tobramycin and related antibiotics. J. Am. Chem. Soc. 96: 3300 3305, 1974

20) Dahlovist, A.: Method for assay of intestinal disaccharidase. Anal. Biochem. 7: 18 25, 1964

21) PENEK, A. \& N. O. SouZA: Purification and properties of baker's yeast trehalase. J. Biol. Chem. 239: $1671 \sim 1673,1964$

22) KALF, G. F. \& S. V. RIEDER: The purification and properties of trehalase. J. Biol. Chem. 230: 691 698, 1958 